\title{
Multi-scale study of the strength and toughness of carbon nanotube fiber materials
}

\author{
Qiu Li ${ }^{\text {a,c }}$, Jian-Shan Wang ${ }^{a}$, Yi-Lan Kang ${ }^{\mathrm{a}, *}$, Ya-Li Li ${ }^{\mathrm{b}}$, Qing-Hua Qin ${ }^{\mathrm{a}}$, Zhu-Lin Wang ${ }^{\mathrm{a}}$, Xiao-Hua Zhong ${ }^{\mathrm{b}}$ \\ a Tianjin Key Laboratory of Modern Engineering Mechanics, School of Mechanical Engineering, Tianjin University, Tianjin 300072, PR China

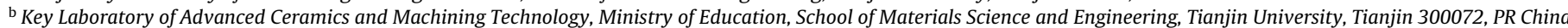 \\ c Tianjin Key Laboratory of High Speed Cutting and Precision Machining, Tianjin University of Technology and Education, Tianjin 300222, PR China
}

\section{A R T I C L E I N F O}

\section{Article history:}

Received 3 August 2011

Received in revised form 14 February 2012

Accepted 5 April 2012

Available online 13 April 2012

\section{Keywords:}

Carbon nanotube fiber

Raman spectroscopy

Multi-scale mechanical property

Constitutive relationship

\begin{abstract}
A B S T R A C T
The control mechanisms of the strength and toughness of carbon nanotube (CNT) fibers are revealed by analyzing the load-bearing and deformation characteristics of multi-scale structures in the fiber under tensile loading. A theoretical model is established to investigate the effect of the multi-scale structures on the strength and toughness of CNT fibers. Based on our previous experimental results on tension with in situ micro-Raman monitoring [Li et al., Nanotechnology 22, 2011], the macro- and micro-mechanical behaviors of the fiber are analyzed. The tensile behaviors of the fiber are correlated with the load-bearing and deformation processes involved in the multi-scale structures in the fiber, such as the nanotube bundle and the thread in microscopic scale, and the CNT in nanoscale. The CNT fiber exhibits high strength and toughness simultaneously depending on the multi-scale structure of the material, the differences in the properties between bundles and threads, and the unique interfaces formed by the tabular geometric configuration of double-walled CNTs. A constitutive relationship for CNT fiber materials is developed to provide information on the role of multi-scale structures on the strength and toughness of fibers. Both strength and toughness of CNT fibers can be enhanced by increasing the volume ratio of bundles to threads, the interfacial shear strength, and the interface slippage friction resistive force among the CNTs.
\end{abstract}

(c) 2012 Elsevier B.V. All rights reserved.

\section{Introduction}

Due to their excellent mechanical properties, carbon nanotubes (CNTs) have been investigated for building high-performance engineering materials since their discovery in 1991. A variety of macroscopic materials assembled using CNTs, including films [1], fibers [2], and blocks [3], have been developed progressively. Among them, CNT fibers generally exhibit high strength and high toughness, which makes them attractive for a wide range of applications in practical engineering. Thus, understanding the underlying mechanisms involved in the development of high strength and toughness in CNT fiber materials is important.

Over the past two decades, much effort has been devoted to investigating the mechanical properties of CNT-based materials in experimental and model characterization. For example, Cheng et al. demonstrated that the winding of single-walled CNT fibers could enhance their material strength to a certain degree through tensile testing [4]. Beyerlein et al. investigated the effects of fiber diameter and gauge length on the statistical strength of CNT fibers

\footnotetext{
* Corresponding author. Tel.: +86 22 87894001; fax: +86 2287894001 .

E-mail address: tju_ylkang@yahoo.com.cn (Y.-L. Kang).
}

[5]. Liu et al. presented a hybrid atom/continuum model to study the nonlinear elastic properties of single-walled CNT bundles as bulk materials [6]; Vilatela et al. developed an analytical model to calculate the theoretical axial strength of CNT fibers [7]. However, the control mechanism for the strength and toughness of CNT fiber materials is still not clear. Generally, the mechanical properties of CNT fibers are strongly affected by fabrication methods and processing techniques because the arrangement of CNTs and their interactions depend significantly on the fabrication process. For example, the strength of CNT fibers spun from CNT suspensions is about $0.15 \mathrm{GPa}$ and their elongation is about $2 \%$ [8]; the strength of fibers drawn out from multi-walled CNT arrays is approximately $0.46 \mathrm{GPa}$ with $10 \%$ elongation [9]; and fibers spun from CNT aerogel formed with chemical vapor deposition (CVD) can reach a tensile strength of up to 1.46-9 GPa. The corresponding elongation ranges from $5 \%$ to $10 \%[2,10,11]$. The studies cited above indicate that multi-scale microstructure and deformation performance are two major control mechanisms of the mechanical properties of CNT fibers. Meanwhile, little attention has been paid in the literature to the toughness of CNT fibers, although strength and toughness are the two most important mechanical properties. Improving the strength and toughness of most CNT-based materials is not possible because of lack of critical knowledge on the underlying 

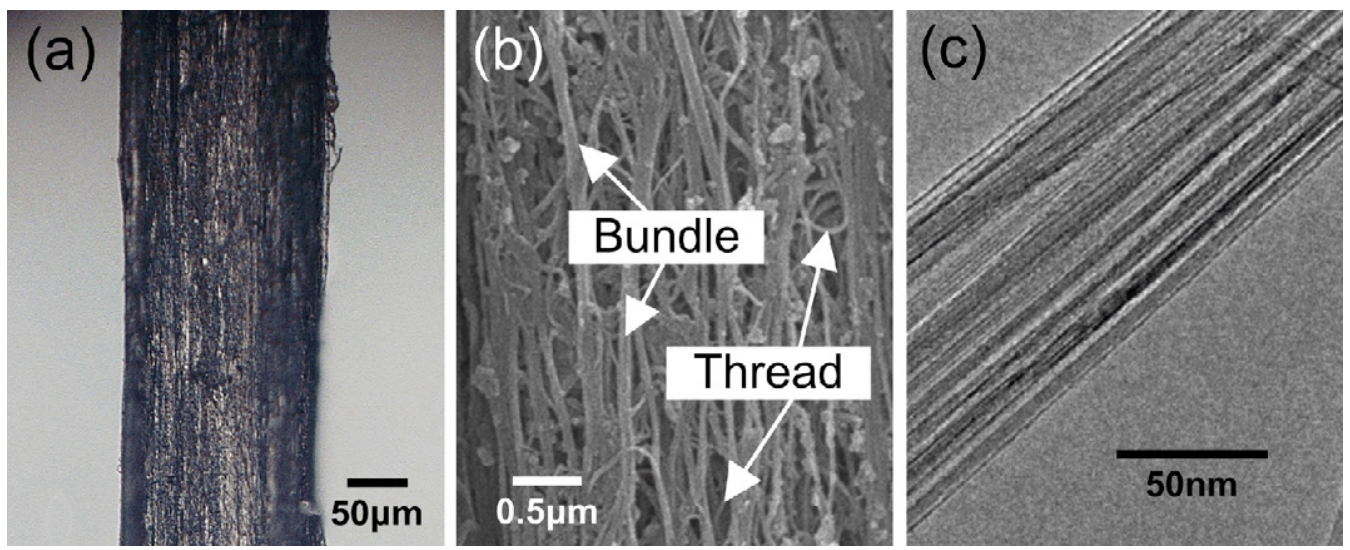

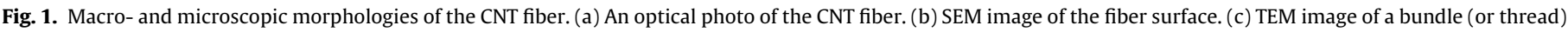
in the fiber.

mechanisms. Therefore, the following questions should be addressed: Why do some CNT fiber materials possess high strength and high toughness simultaneously? How are the strength and toughness of the materials affected by their microstructures? How can we effectively characterize the strength and toughness of these CNT fibers?

In our previous work [12], tensile tests coupled with in situ Raman detection have been carried out for CNT fibers spun from CNT aerogel formed with CVD. The individual CNTs deform elastically without obvious damage or bond breakage. The yield and fracture of fibers can be due to the slippage among the CNTs. In this work, the strength and toughness of CNT fibers are studied at multi-scale levels. Based on the macroscopic tension tests and in situ micro-Raman detection results and the microstructure characteristics of the fibers observed, the load-bearing and deformation processes of multi-scale structures in the fibers under tension are analyzed to provide an insight into the control mechanisms of the strength and toughness of CNT fibers. More importantly, a constitutive relationship for CNT fiber materials is developed using the analysis described. The constitutive relationship is then used to investigate the influence of the multi-scale structures on the macroscopic strength and toughness of the fibers.

\section{Mechanisms of the strength and toughness of CNT fibers}

The CNT fiber materials are spun from CNT aerogel formed by CVD [13]. Macro and micro morphologies of the fiber are shown in Fig. 1. The fiber has a multi-scale structure: the fiber itself is in macroscopic scale, the nanotube bundle and thread in microscopic scale, and the CNT in nanoscale. The macroscopic fiber (Fig. 1(a)) contains relatively thick and straight bundles, as well as fine, flexible threads (Fig. 1(b)). The bundles preferentially align along the fiber axis. The threads are distributed among the bundles and combined. Both the bundles and threads are formed by piling double-walled CNTs with a collapsed section (Fig. 1(c)). Further information on this process can be found elsewhere [13].

\subsection{Tension experiment of CNT fibers coupled with Raman detection}

The uniaxial tension of the CNT fiber is measured by a precise micro-loading device. The fibers are stuck onto the center-hollow paper cards and then mounted in the micro-loading device with cards. The fiber gauge lengths are fixed to $5 \mathrm{~mm}$, and the average strain rate is $0.7 \% \mathrm{~min}^{-1}$. During the application of uniaxial tension to the fibers, the spectrum data are collected by a Renishaw InVia Raman spectroscope. Detailed information can be found in our

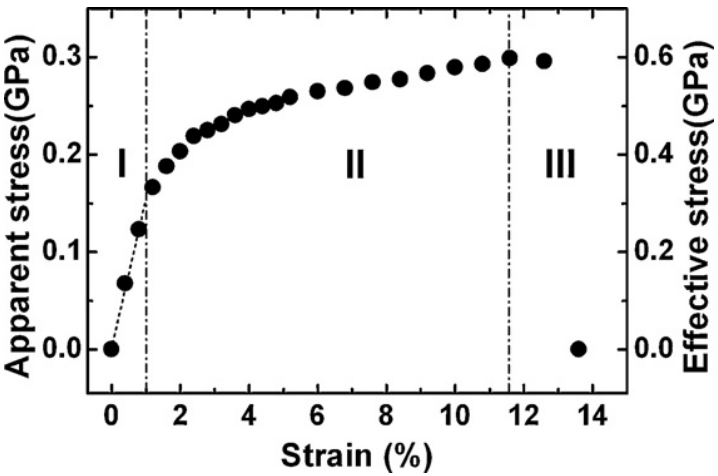

Fig. 2. Change in tensile stress of CNT fiber with strain.

previous work [12]. The macroscopic stress-strain curve and the $G^{\prime}$ Raman band information of the fibers are given in Figs. 2 and 3, respectively.

\subsection{Mechanisms of the strength and toughness of CNT fibers}

The deformation process of the fibers includes three stages, namely, the elastic stage I (strain $<1 \%$ ), the hardening stage II $(1 \% \leq$ strain $\leq 11.5 \%)$, and the damage-fracture stage III (strain $>11.5 \%$ ), as shown in Fig. 2, where the fiber strain is obtained from the ratio of its deformation quantity to gauge length. The fiber deformation quantity is read out from the microloading device. The apparent wall thicknesses and circumferences of the fibers are obtained from the scanning electron microscope (SEM) images of the cross-sections of the fibers. The apparent Young's modulus, yield limit $\left(\sigma_{0.2}\right)$, and strength limit of the CNT fiber are estimated as $15.6,0.14$, and $0.3 \mathrm{GPa}$, respectively. These data are based on the SEM images where a large space inside the fiber walls exists. Thus, the volume (or area) occupied by the space should be excluded from the total volume (or area) when calculating the effective mechanical properties based on the actual cross-sectional loaded area. According to the SEM observations, the estimated volume fraction of CNTs inside the fiber walls is $\leq 50 \%$. Thus, the effective calculated Young's modulus, yield limit and strength limit of the fibers should be at least twice of the values mentioned above, namely, 31.2, 0.28, and $0.6 \mathrm{GPa}$, respectively. The elongation of the fibers given in the experiment is approximately $11 \%$. All these data indicate that the CNT fibers exhibit both outstanding strength and relatively high toughness simultaneously. 

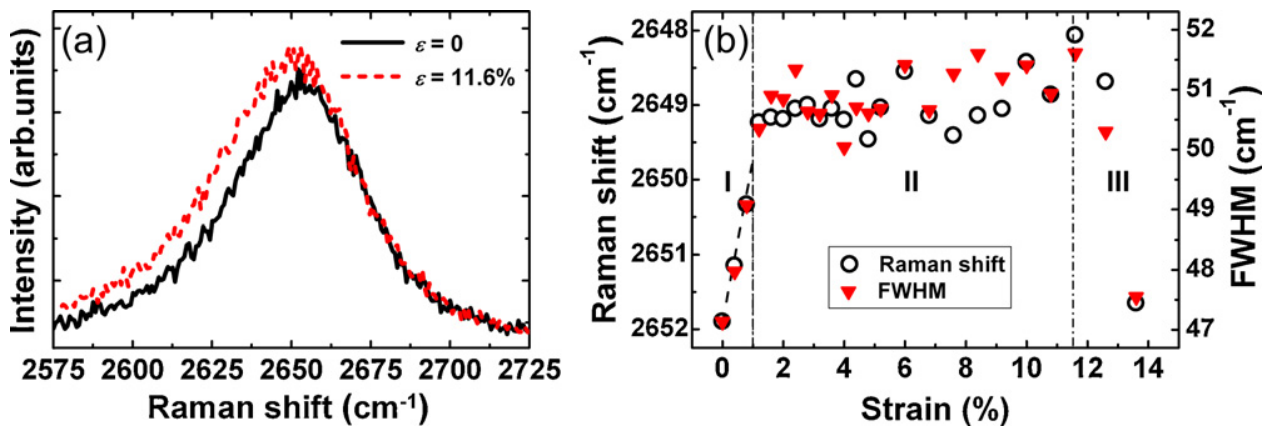

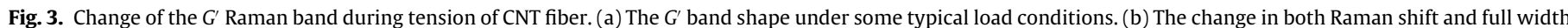
at half-maximum (FWHM) of the $G^{\prime}$ band with fiber strain.

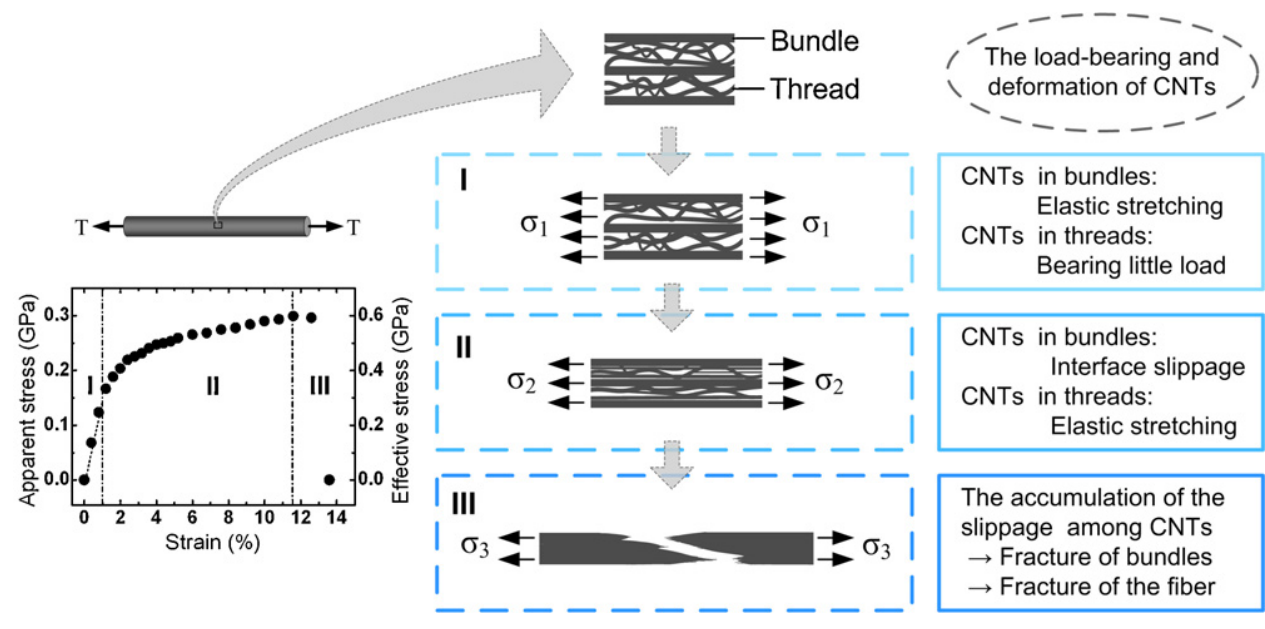

Fig. 4. Load-bearing and deformation characteristics of multi-scale structures in CNT fibers under tensile loading.

Based on the information from the $G^{\prime}$ Raman band, a physical model for the load-bearing and deformation characteristics of multi-scale structures in fibers (Fig. 4) is developed by integrating the microstructure characteristics of the fiber and the features of its macroscopic mechanical behaviors during the tensile process. The analysis of the relationship between the deformation evolution of the multi-scale structures in the fiber and its macroscopic mechanical behaviors, as well as the control mechanisms of the strength and toughness of the CNT fiber, are described in the succeeding paragraphs.

In elastic stage I, the main load is first borne by the bundles inside the fiber, with linearly increased elastic deformation, when the external load is applied gradually to the fibers. The elastic deformation of the CNTs in bundles also increases linearly with the external load. Curly threads are gradually stretched, and the internal CNTs bear little load. The macroscopic stress of the fiber increases linearly with the strain. During this stage, the average elastic deformation of the CNTs inside the fiber increases linearly with the fiber strain, and consistently with the variation of the Raman shift of the $G^{\prime}$ band (Fig. 3(b)). In addition, the dominant load is shared by the CNTs inside the bundles, which increases with the increasing external loading. Only small part of the load is shared by the CNTs inside the threads, which changes slightly. As a result, the CNTs inside the fiber bear load unevenly, with continuously increasing differences between the loads borne by the bundles and the threads. The continuously increasing $G^{\prime}$ band FWHM with the fiber strain is consistent with the previous observation.

Once the stress on the interfaces among the CNTs inside the bundles exceeds the interfacial shear strength, slippage will occur at those interfaces. Slippage may also occur between the internal and external walls of double-walled CNTs. Although the increment of elastic deformation of the CNTs inside the bundles is very small during the process of slippage, slippage can induce large plastic deformation on the bundles. Meanwhile, some threads are gradually pulled straight and can bear now part of the load. Thus, the CNTs inside the threads bear increasing load with an increase in elastic deformation. At this stage, the macroscopic stress of the fibers increases very slowly with the fiber strain, and the fibers show strain hardening, as observed in hardening stage II. The existence of this deformation feature can be attributed to the special bundle and thread structures of the CNT fibers. Simply put, the threads will gradually participate more actively in load bearing when sliding deformation occurs in the bundles. On the other hand, the tabular geometric configuration of the double-walled CNTs may cause relatively strong slippage friction resistive force. At this stage, little change occurs in the axial deformation of the CNTs inside the bundles, so both the Raman shift and the FWHM of the $G^{\prime}$ band change slowly, as shown in Fig. 3(b). Moreover, during the tensile process the threads are gradually pulled straight and the CNTs inside the threads are aligned to the loading direction, as observed by the increasing trend of the $G^{\prime}$ band intensity shown in Fig. 3(a).

During damage and fracture stage III, the continuous accumulation of slippage damage on the interfaces among the CNTs inside the bundles causes the CNTs to be separated from each other and the bundles to be fractured, although more threads are involved in load bearing. Expansion of the fracture nucleation will induce gradual damage to the fiber. 


\section{Constitutive relationship of CNT fiber materials}

Based on the load-bearing and deformation process of multiscale structures in CNT fibers described above, a constitutive equation of the CNT fiber materials is developed to represent mathematically the macroscopic physical relationships of the CNT fibers. Considering their microstructures and deformation characteristics, CNT fiber materials can be viewed as a special fiber consisting of two load-bearing materials, the thread material and the bundle material. Of the two materials, the thread material is stretched relatively easily. Therefore, its stress always increases slowly with strain when it experiences tensile loading. By contrast, the bundle material with a relatively large Young's modulus generally experiences elastic deformation first under tensile loading. Once its stress exceeds a critical value, the bundle material enters a plastic deformation stage.

The fiber can be taken as a quasi-one-dimensional material and it should satisfy the equilibrium and deformation compatibility conditions Eqs. (1) and (2), namely, the strain of the fiber is equal to the equivalent strain of the thread material and the equivalent strain of the bundle material, whereas the total stress of the fiber is equal to the sum of the stresses on both the thread material and the bundle material:

$d \varepsilon=d \varepsilon_{t}=d \varepsilon_{b}$,

$d \sigma=V_{t} d \sigma_{t}+V_{b} d \sigma_{b}$,

where $d \varepsilon$ and $d \sigma$ are the effective strain increment and the effective stress increment of the fiber, respectively; $d \sigma_{t}, d \sigma_{b}, d \varepsilon_{t}$, and $d \varepsilon_{b}$ are the equivalent stress and equivalent strain increments of the thread material and the bundle material, respectively; $V_{t}$ and $V_{b}\left(=1-V_{t}\right)$ represent the equivalent volume fractions of the thread material and the bundle material, respectively.

According to its deformation characteristics, the thread material is approximately a linear elastic material whose stress-strain relation can be defined as follows:

$\sigma_{t}=E_{t} \varepsilon_{t}$,

where $E_{t}$ is the equivalent Young's modulus of the thread material. $E_{t}$ depends on the degree of bending of the threads, the compactness of the threads, and the interfacial properties among the threads.

The mechanical properties of the bundle material are described by the Ramberg-Osgood (RO) power hardening model [14,15]. Its stress-strain relation can be written as follows:

$\frac{\varepsilon_{b}}{\varepsilon_{b 0}}=\frac{\sigma_{b}}{\sigma_{b 0}}+C\left(\frac{\sigma_{b}}{\sigma_{b 0}}\right)^{n}$,

where $\sigma_{b 0}, \varepsilon_{b 0}=\sigma_{b 0} / E_{b}$ are the yield stress and yield strain of the bundle material, respectively; $E_{b}$ is the Young's modulus of the bundle material, and generally $E_{b} \gg E_{t}$. $C$ and $n(n>1)$ are two dimensionless constants that represent the hardening coefficient and hardening index of the bundle material, respectively. $C$ is related to the interfacial shear strength among the CNTs. The smaller the constant $C$, the greater the interfacial shear strength among the CNTs. $n$ is related to the friction resistive force of interface slippage among the CNTs, in that the smaller the constant $n$, the greater the friction resistive force of interface slippage among the CNTs.

Using Eqs. (1)-(4), a constitutive equation of the CNT fiber material can be obtained as follows:

$\frac{\varepsilon}{\varepsilon_{0}}=\frac{1}{V_{b}}\left(\frac{\sigma}{\sigma_{0}}-\frac{V_{t} E_{t} \varepsilon}{E_{b} \varepsilon_{0}}\right)+\frac{C}{V_{b}^{n}}\left(\frac{\sigma}{\sigma_{0}}-\frac{V_{t} E_{t} \varepsilon}{E_{b} \varepsilon_{0}}\right)^{n}$,

where $\varepsilon / \varepsilon_{b 0}$ and $\sigma / \sigma_{b 0}$ are dimensionless strain and stress, respectively; $\varepsilon_{0}$ and $\sigma_{0}$ are the yield strain and yield stress of the fiber,
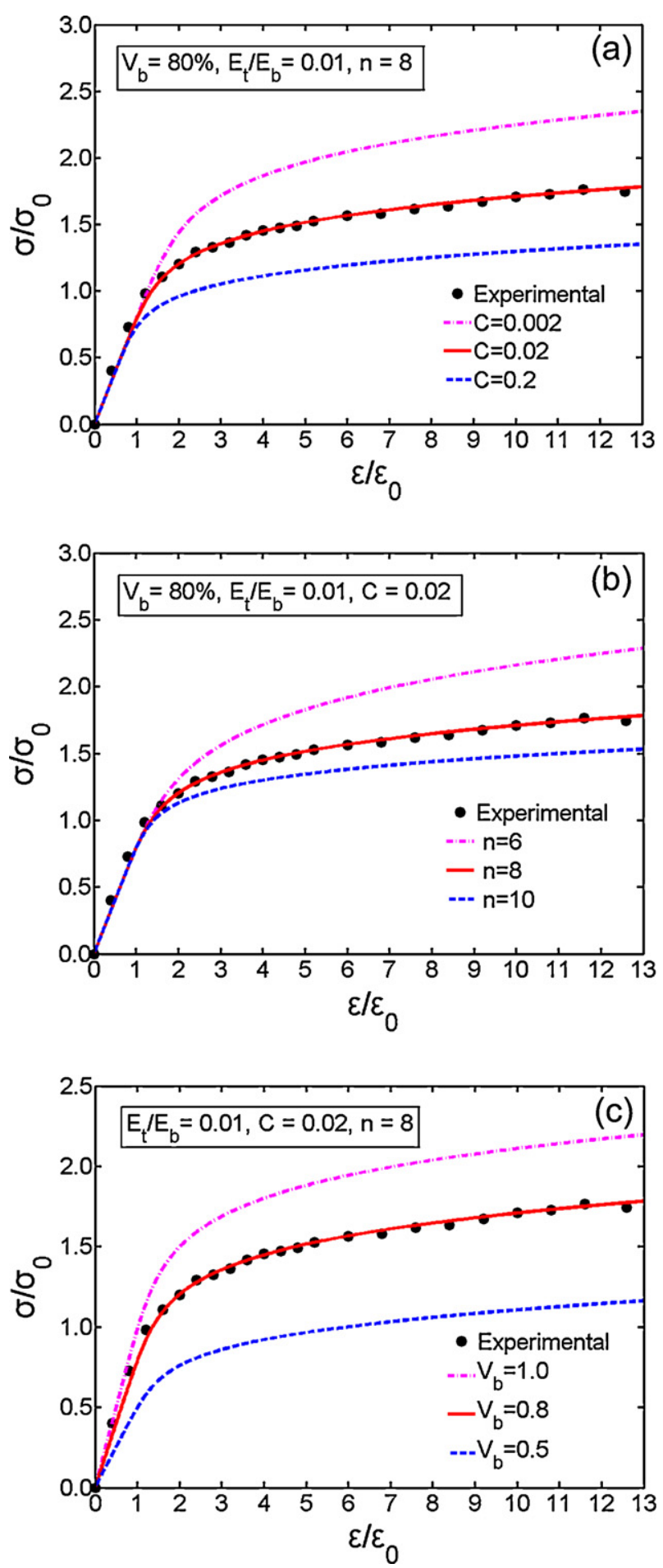

Fig. 5. Constitutive relationship of the CNT fiber material. (a) Constitutive relationship with different hardening coefficient $C$. (b) Constitutive relationship with different hardening indices $n$. (c) Constitutive relationship with different volume ratios of bundles to threads. (For interpretation of the references to color in this figure legend, the reader is referred to the web version of this article.)

respectively; and $\varepsilon_{0}=\varepsilon_{b 0}, \sigma_{0}=\sigma_{b 0} . E_{t} / E_{b}$ is the equivalent Young's modulus ratio of the thread material to the bundle material.

The material parameters appearing in Eq. (5) can be determined by fitting the tensile experiment data of the fiber materials. For the fiber materials used in this work, the volume fractions of the bundle material and thread material, namely, $V_{b}$ and $V_{t}$, are $80 \%$ and $20 \%$, respectively, according to the SEM images. $E_{b} \gg E_{t}$, so $E_{t} / E_{b}$ is taken 
as 0.01 . By least square fitting the experimental data shown in Fig. 2 with Eq. (5), the hardening coefficient $C$ and hardening index $n$ are obtained to be 0.023721 and 7.8177 , respectively. Rounding off $C$ and $n$ yields the values 0.02 and 8 , respectively. The fitted curve is shown in Fig. 5 in red (the middle curve in the figure). Therefore, the constitutive relationship of the CNT fiber materials used in this study can finally be written as follows:

$\frac{\varepsilon}{\varepsilon_{0}}=1.247 \frac{\sigma}{\sigma_{0}}+0.119\left(\frac{\sigma}{\sigma_{0}}-0.002 \frac{\varepsilon}{\varepsilon_{0}}\right)^{8}$.

Using of the constitutive Eq. (5), the effects of the material's microstructure on the strength and toughness of the CNT fibers are further studied. Fig. 5(a) and (b) shows the effects of interfacial shear strength and friction resistive force of interface slippage on the macroscopic mechanical properties (strength and toughness) of the CNT fibers, respectively, when the volume ratio and elastic properties of the bundles and threads remain unchanged. It can be seen from Fig. 5(a) that the greater the interfacial shear strength among the CNTs (the lower the hardening coefficient $C$ ), the higher the yield limit of the fiber, meaning the CNT fibers will have higher strength. Furthermore, Fig. 5(b) illustrates that the greater the friction resistive force of interface slippage among the CNTs (the lower the hardening index $n$ ), the higher the degree of strain hardening of the fiber. In addition, Fig. 5(c) reveals that the greater the volume ratio, the better the strength and toughness of the fiber. When the volume ratio increases, the Young's modulus and strength toughness properties of the fiber are improved. This is because the bundles bear most of the load during the elastic stage of the fiber.

\section{Conclusion}

For continuous CNT fibers spun from CVD aerogel, based on tensile and in situ Raman experimental results, the multi-scale structure deformation characteristics and evolution related with the macroscopic mechanical behaviors are analyzed. It is deduced that the elastic deformation of the fibers is mainly attributable to the elastic stretching of the CNTs inside the bundles, and the deformation in the hardening stage is caused mainly by slippage at the interfaces among CNTs inside the bundles. The CNT fiber can simultaneously possess both high strength and toughness, depending on the significant multi-scale structure of the material, the differences in the properties between bundles and threads, and the unique interfaces formed by the tabular geometric configuration of double-walled CNTs.

A new constitutive equation is developed to characterize the macroscopic mechanical properties and the influence of the multiscale structural parameters on the strength and toughness of CNT fibers. The strength and toughness of the CNT fiber can be enhanced by increasing the volume ratio of bundles to threads, the interface shear strength, and interface slippage friction resistive force among the CNTs. Our investigation presents initial insight into the mechanisms of the high strength and toughness of CNT fibers, and provides the theoretical basis for improvement of the manufacturing technology to enhance their mechanical performance.

\section{Acknowledgments}

We acknowledge the financial support from the National Basic Research Program of China (No. 2012CB937500), the National Natural Science Foundation of China (No. 11002097) and the Key Grant of Chinese Ministry of Education (No. 309010).

\section{References}

[1] R.H. Baughman, C. Cui, A.A. Zakhidov, Z. Iqbal, J.N. Barisci, G.M. Spinks, G.G. Wallace, A. Mazzoldi, D.D. Rossi, A.G. Rinzler, O. Jaschinski, S. Roth, M. Kertesz, Science 284 (1999) 1340.

[2] Y.L. Li, I.A. Kinloch, A.H. Windle, Science 304 (2004) 276.

[3] X.C. Gui, J.Q. Wei, K.L. Wang, A.Y. Cao, H.W. Zhu, Y. Jia, Q.K. Shu, D.H. Wu, Adv Mater. 22 (2010) 617.

[4] T.W. Cheng, W.K. Hsu, Appl. Phys. Lett. 90 (2007) 123102

[5] I.J. Beyerlein, P.K. Porwal, Y.T. Zhu, K. Hu, X.F. Xu, Nanotechnology 20 (2009) 485702.

[6] J.Z. Liu, Q.S. Zheng, L.F. Wang, Q. Jiang, J. Mech. Phys. Solids 53 (2005) 123

[7] J.J. Vilatela, J.A. Elliott, A.H. Windle, ACS Nano 5 (2011) 1921.

[8] B. Vigolo, A. penicaud, C. Coulon, C. Sauder, R. Pailler, C. Journet, P. Bernier, P. Poulin, Science 290 (2000) 1331.

[9] M. Zhang, K.R. Atkinson, R.H. Baughman, Science 306 (2004) 1358.

[10] M. Motta, Y.L. Li, I. Kinloch, A. Windle, Nano Lett. 5 (2005) 1529.

[11] K. Kozio, J. Vilatela, A. Moisala, M. Motta, P. Cunniff, M. Sennett, A. Windle, Science 318 (2007) 1892.

[12] Q. Li, Y.L. Kang, W. Qiu, Y.L. Li, G.Y. Huang, J.G. Guo, W.L. Deng, X.H. Zhong, Nanotechnology 22 (2011) 225704.

[13] X.H. Zhong, Y.L. Li, Y.K. Liu, X.H. Qiao, Y. Feng, J. Liang, J. Jin, L. Zhu, F. Hou, J.Y. Li, Adv. Mater. 22 (2010) 692.

[14] J.W. Hutchinson, J. Mech. Phys. Solids 16 (1968) 13

[15] W. Ramberg, W.R. Osgood, Technical Note No. 902, National Advisory Committee for Aeronautics, Washington, DC, 1943. 